\title{
26 Research Square \\ Refractive Index Sensor Based on Double D-Shaped Using Fiber Bragg Grating
}

chetna upadhyay ( $\square$ chetna1876@gmail.com )

Punjab Engineering College: PEC University of Technology

Divya Dhwan

Punjab Engineering College: PEC University of Technology

\section{Research Article}

Keywords: Double D-shaped fiber, fiber grating, fiber optic sensors, refractive index sensor.

Posted Date: March 2nd, 2022

DOI: https://doi.org/10.21203/rs.3.rs-1143953/v1

License: (9) This work is licensed under a Creative Commons Attribution 4.0 International License. Read Full License 


\section{Abstract}

FBG based refractive index sensor are widely used in sensing applications such as structural health monitoring, oil exploration, refractive index measurement of chemical solutions, etc., owing to benefits of FBG such as small size, real time monitoring, high resolution, resistant to high temperature and various chemicals, etc. In this paper, simple and high sensitive method employing a double D-shaped FBG (DDSF) for refractive index (RI) measurements of glucose solution is proposed and investigated. The operating principle is based on the exploitation of the evanescent wave interaction of the propagating light with the surrounding external environment, resulting in shift of Bragg wavelength. Wavelength shift is measured, when the refractive index of external medium changes from 1.33 to 1.43 approximately for glucose solution with varying concentrations, exhibiting a good linear relationship between them. By changing the depth of the D-shaped groove, the sensor's sensitivity can optimize. A high sensitivity of $47.37 \mathrm{~nm} / \mathrm{RIU}$ is achieved, which indicates that proposed sensor has provided high sensitivity and linearity.

\section{Introduction}

In the field of fiber optics, refractive index sensors have been widely investigated because of their potential applications in chemical, biological, and food industries. Such sensors have many advantages over conventional electrical sensors like faster response, immune to electromagnetic interference, lesser cost, and higher sensitivity [1][2][3][4]. Generally, fiber Bragg grating is commonly used to measure temperature, stress, pressure, refractive index, humidity, etc. [5][6][7][8][9][10][11]. Various optical elements such as FBGs (fiber Bragg gratings) [12], microfiber interferometer [13], multimode interference structures [14], etc. are used to measure refractive index of different chemical solutions, etc. Amongst them FBG is preferred over other technologies because it can be used for remote distribution sensing, fast interrogation, etc. For remote distributed sensing fiber Bragg grating is suitable to measure refractive index but before specific modifications, it has larger temperature cross-sensitivity [15]. FBG based refractive index sensors are admired choices, when there is a need to deal with the quality check of certain liquids or materials, for structural monitoring applications like structure bending, etc., to measure $\mathrm{pH}$ values of certain solutions, to check the toxicity of the different liquids in industries. A high sensitivity using ordinary FBG is not possible and some modifications are need to be done like improvision of structure, usage of different types of gratings, coatings of different materials, etc. can be used.

Modifications can be done in structures, grating type and different materials. Structures such as Dshaped [16], thinned shaped [17], micro-structured FBG [18], hollow suspended core fiber [19], helical microfiber Bragg grating [20], spherical-shape structure [15], thinned cladding structure [21], etc. are used to improve the sensitivity of refractive index sensor. To make such special structures different processes like mechanical or chemical etching, tapering [22], etc. are used. After etching cladding part of sensor, sensitivity is improved as more evanescent waves are formed but with the etching of cladding mechanical strength of the sensor is also reduced which results in lower durability. Different types of gratings like uniform grating [10], long-period grating [23], chirped grating [24], tilted grating [25], are also widely used but amongst them long period are widely used. By using long period grating sensitivity is 
higher but coupling losses are also there. Coating materials like reduced graphine oxide [26], polymer [27], titanium oxide [28], silicon oxide[29], negative refractive index modification line [21], etc. have been used in refractive index sensor. Specially designed structures with different types of the grating such as hollow eccentric optical fiber(HEOF) [8], SMF- TCF-SMF (STCS) results with Mach Zehnder interferometer [30], Mach-Zehnder fiber interferometers [31] with long period fiber gratings, etc.

To improve the sensitivity etched structures are widely used. Using etching when cladding of fiber is etched such that one side of fiber looks like $D$ in shape, higher sensitivity is achieved than that of ordinary sensor [32]. With an increase in etching the sensitivity of refractive index sensor is also increased proportionally [17][21]. Consequently, to improve the sensitivity, in novel double D-shaped refractive index sensor, etching has been done from opposite side in D-shaped fiber resulting in $2 \mathrm{D}$-shapes in refractive index sensor. This proposed novel double D-shaped fiber, whose structure is cascaded with an FBG (fiber Bragg grating) inscribed inside the single mode fiber is used for RI sensing of glucose solution with different concentrations. By observing the shift of Bragg wavelength of structure of double D-shaped fiber, sensitivity of sensor can be obtained for the refractive index ranging from 1.33 to 1.43 approximately. For D-shaped fiber the achieved sensitivity is of $35.26 \mathrm{~nm} / \mathrm{RIU}$ and for double D-shaped refractive index sensor the achieved sensitivity is of $47.37 \mathrm{~nm} / \mathrm{RIU}$. As the depth of etching is increased the sensitivity of the sensor is also increased as stronger evanescent field is experienced by cladding modes but there is decrement in the mechanical strength and durability of sensor. Also, the results of Dshaped fiber and double D-shaped fiber are compared with the existing work in terms of sensitivity of refractive index. The proposed sensor has characteristics of compact structure, simple configuration, and higher sensitivity, which provides potential applications in RI measurement.

\section{li. Design And Principal Of Double D-shaped Fiber}

\section{a) Principle of FBG sensor}

A fiber Bragg grating is a periodic deviation of refractive index in core along the length of optical fiber by exposing its core to an intense optical interface pattern in presence of ultraviolet (UV) light [33]. Being a fiber optic sensor, a Bragg grating has all the advantages usually attributed to significances, such as low loss relative to the fiber length, immunity to electromagnetic and radio frequency interference, small size and weight, intrinsically safe operation in environments characterized by hazardous materials, high sensitivity and long-term reliability. Fiber Bragg grating technology reveals an inherent serial multiplexing capacity and an ability to provide absolute measurements without the need for referencing. This makes it the natural alternative to conventional electrical sensing technologies. When the phase- matching condition is satisfied, the contributions of reflected light from each grating plane add constructively in the backward direction to form a back-reflected peak with a center wavelength defined by the grating parameters [4]. The reflected wavelength which is also known as Bragg wavelength can be measured using Bragg condition as expressed in Eq. (1):

$\lambda_{\mathrm{FBG}}=2 \mathrm{n}_{\mathrm{eff}} \wedge \ldots(1)$ 
Where, neff is the effective index and $\Lambda$ is the period of grating. Shift in Bragg wavelength $(\Delta \lambda F B G)$ can be expressed as the twice of product of change of effective refractive index ( $\Delta$ neff) of the surrounding of the sensor and grating period of the FBG inside the sensor as shown in Eq. (2). Therefore, Bragg wavelength shift can be expressed as in Eq. (2) [34]:

$\Delta \lambda \mathrm{FBG}=2 \Delta \operatorname{neff} \wedge$

Thus, FBG sensor for detection of the environmental refractive index change is based on the Bragg wavelength shift.

\section{b) D-shaped fiber FBG based refractive index sensor}

The structure of D-shaped fiber (DSF) as illustrated in Fig. 1, is a combination of two single mode fibers with grating inscribed in one of the SMF. In D-shaped refractive index sensor, cladding part is etched from one side. When incident light encounters D-shaped fiber with fundamental mode, from core mode a segment of light will be coupled with modes of higher order of cladding in D-shaped fiber. A similar study has been done in [32], where D-shaped refractive index sensor is used to find sensitivity of glycerin solution. Refractive index sensor uses the total internal reflection (TIR) concept, where the light transmits through the fiber is frequently reflecting from interface of cladding-core in a very lossless manner [11]. For reflecting light at angle which is near to the critical angle, a significant portion of the power (a decayed electric field) extends into the cladding or medium which surrounds the core, known as the evanescent wave, extends only to a short distance from the interface, with power dropping exponentially with distance [35]. The strength of these evanescent fields is also affected by environmental refractive index variation around them. Bragg grating reflection or transmission properties have to be strongly affected to measure the change in refractive index of D-shaped fiber efficiently. Different types of evanescent fields are generated by the different modes which are propagating through the fiber, which leads to radiation of optical field outside the core of the fiber. As the high order of modes increase, the generation of corresponding evanescent field will also increase. Consequently, in evanescent areas modes of higher orders have larger optical power distribution resulting in more sensitivity of the refractive index sensor.

Both core and cladding mode will propagate in the D-shaped fiber and this region will act as a channel for light and matter interaction. Modes of cladding will interfere with core modes because of different modal phases and in lead out SMF they get interfere resulting in making an interferometer for all fiber modals. A relatively stronger evanescent field is formed outside the $D$-shaped fiber because of the etched region. As the RI sensor is etched partially from the cladding part, a stronger evanescent field is experienced by cladding modes in D-shaped fiber outside the cladding of sensor. When there is a deviation in the concentration of glucose, the RI of sensor will also change which results in shift of Bragg's wavelength traveling through D-shaped fiber.

\section{c) Double D-shaped fiber FBG based refractive index sensor}


To improve the sensitivity, more cladding part of the sensor is needed to be etched. Therefore, cladding of the D- shaped FBG sensor etched from the opposite side of etched section of D-shaped fiber, which results in structure of double D-shaped FBG sensor as shown in Fig. 2. At the interference of double Dshaped fiber and its external environment, the generated electric field is higher than that of DSF, as a wider area is etched in double D-shaped. The strength of these evanescent fields is more affected by environmental refractive index variation for the double D- shaped fiber. Consequently, in evanescent areas modes of higher order have larger optical power distribution resulting in more sensitivity of the refractive index sensor. When incident light encounters double D-shaped fiber from core mode a segment of light coupled with modes of higher order of cladding in double D-shaped fiber. Both core and cladding mode will propagate through the double D-shaped fiber and this region will act as a light and matter interaction channel. More no of modes of cladding will interfere with core modes because of different modal phases and in lead out SMF they get interfere resulting in the making of the interferometer for all fiber modal. When there is a deviation in concentration of glucose solution with refractive index ranging from 1.33$1.43, \mathrm{RI}$ of sensor will also deviate and this results in a shift in reflected Bragg wavelength of transmission spectrum traveling through double D-shaped fiber.

\section{lii. Results And Discussion}

The designing and simulation of double D-shaped fiber based refractive index sensor is carried out on gratingMOD tool of optical software R-Soft CAD. Using gratingMOD, the refractive index sensitivity of Dshaped and double D-shaped refractive index sensor are measured for glucose solution. Using parameters as shown in table 1 , a structure with core diameter of $13.5 \mu \mathrm{m}$ and cladding diameter of 139 $\mu \mathrm{m}$ is used in designing of $\mathrm{D}$-shaped and double $\mathrm{D}$-shaped fiber. Here, grating type is volume index with structure type as fiber and grating period is of 0.5 . Background refractive index is considered as refractive index of glucose solution which is required to be sensed. Delta for core is 0.028 and for cladding is 0.02 , when concentration of glucose solution is considered as 50\%. Depth of etching for D-shaped groove is of $30 \mu \mathrm{m}$ from each side. Free space wavelength considered RI sensor is of $1.45 \mu \mathrm{m}$. Fiber Bragg grating is inscribed in fiber from length (I) $0 \mu \mathrm{m}-1000 \mu \mathrm{m}$ in Z-direction.

For increased concentration of glucose in solution within a range of $0 \%-50 \%$ in water, RI changed from 1.33 to 1.43 . When the concentration of glucose in water is $0 \%$, corresponding refractive index is 1.33 and with the increased glucose concentration in water i.e. upto $10 \%$, its refractive index is also increase to 1.35. Similarly, for refractive index 1.43 the concentration of glucose in water is reached to $50 \%$. When cladding part is etched, sensitivity of the sensor increases with the increase of depth of etching in cladding. The ratio of difference between the shift in wavelengths of glucose solution at $0 \%$ concentration and $50 \%$ concentration to the difference of corresponding refractive index will result in sensitivity of DSF sensor. For refractive index of glucose solution ranging between 1.33-1.43 approximately, the obtained sensitivity is $35.26 \mathrm{~nm} / \mathrm{RIU}$. All the shifted wavelengths are represented in the Fig. 4. 
As double D-shaped fiber has stronger evanescent field outside area of the cladding which results in stronger interference influenced by the SRI (surrounding RI) than D-shaped fiber. The core of double Dshaped fiber is exposed more than that of D-shaped fiber and is bordered by silica cladding consequently it is higher in sensitivity to refractive index of surrounding external environment. Figure 5 represents the power spectrum response for reflection using double D-shaped FBG based refractive index sensor.

When the concentration of glucose is increased i.e. from 1.33-1.43 approximately, a blue shift in wavelength is observed in for both D-shaped fiber and double D-shaped fiber. When the concentration of glucose in solution is increased approximately $10 \%$ in water, RI changed from 1.33 to 1.35 and a shift of wavelength for double D- shaped fiber is achieved. In similar way, when the concentration of glucose is increased upto $50 \%$ Bragg's wavelength is shifted further. This shift results in sensitivity of $47.37 \mathrm{~nm} / \mathrm{RIU}$ for refractive index ranging from 1.33 to 1.43 for different concentration of glucose solution using double D-shaped refractive index sensor. At the interface of external environment and double D-shaped fiber, a more evanescent waves (decaying electric field) were generated than D-shaped fiber because of more exposure of the core after etching. For surrounding RI of 1.33, guided mode is confined to core region resulting in weak evanescent field. But as the SRI is increased by increasing in concentration of glucose solution guide mode is less confined to core and results in strong evanescent field and more efficient interaction with surrounding medium. Consequently, light spectrum travelling through the double Dshaped fiber imposes higher shift in Bragg's wavelength for SRI 1.43 than DSF for same surrounding refractive index. A comparison of both type of D-shaped based refractive index sensor is shown in fig.6. In fig. 7, the shift in wavelength for different values of refractive index with different concentrations of glucose solution is represented. From fig. 7, it is also evident that the shift in D-shaped fiber is lesser as compared to the shift in double D-shaped fiber. For smaller value of the refractive index there is smaller increment in the wavelength shift, while for the higher values, there is more shift of wavelength for double D-shaped fiber.

The comparison of sensitivity of proposed double D-shaped FBG based refractive index sensor and Dshaped FBG based sensor with existing work has been done. The achieved sensitivity for D-shaped fiber is $35.26 \mathrm{~nm} / \mathrm{RIU}$ and for DDSF is $47.37 \mathrm{~nm} / \mathrm{RIU}$ for 1.33 to 1.43 range of refractive index for glucose solution. Whereas, in previous work achieved sensitivity is $-31.79 \mathrm{~nm} / \mathrm{RIU}$ for glycerin solution for refractive index ranging between 1.330-1.428. The sensitivity of D-shaped fiber is lower than that of double D-shaped, which clearly shows that with the increase in depth of etching sensitivity of refractive index sensor will also increase.

\section{Iv. Conclusion}

Design of a D-shaped fiber and double D-shaped fiber has been proposed for sensing the glucose solution with refractive index ranging from 1.33-1.43. The etched section of D-shaped fiber and double D-shaped fiber is used for sensing refractive index. Because of the etched cladding, the resulting interference is affected by the surrounding refractive index, and become more sensitive to the external refractive index. Sensitivity of $35.26 \mathrm{~nm} / \mathrm{RIU}$ and $47.37 \mathrm{~nm} / \mathrm{RIU}$ is achieved for both D-shaped fiber and 
double D-shaped fiber RI sensors respectively. Such a sensors are compact and small in structure, therefore, can be used for refractive index sensing in medical and bio-medical applications.

\section{Declarations}

\section{Acknowledgement}

Chetna is a Master's student at Punjab Engineering College (Deemed to be University), Chandigarh. She received her $B E$ degree in electrical and electronics engineering from Technology Education Research Integrated Institute, Kurukshetra University, in 2014. Her area of research includes optical sensing and communication.

Divya Dhawan is an associate professor in the Electronics and Communication Engineering Department at Punjab Engineering College (Deemed to be University), Chandigarh. She received her PhD in optical communication in 2017. Her research interests include optical OFDM, passive optical networks, digital system design, and optical sensors. She has a number of publications in optical communication. She is member of various technical societies, such as IEEE (Electronic Devices), ISTE, and IEI.

\section{Author contribution}

All authors contributed equally.

\section{References}

[1] F. Urban, J. Kadlec, R. Vlach, and R. Kuchta, "Design of a pressure sensor based on optical fiber Bragg grating lateral deformation," Sensors, vol. 10, no. 12, pp. 11212-11225, 2010, doi: 10.3390/s101211212.

[2] M. A. Riza, F. M. Foong, Y. I. Go, and C. K. Thein, “Development of sustainable FBG sensor for environmental monitoring through vibration energy harvesting," 2019 IEEE Int. Conf. Sensors Nanotechnology, SENSORS NANO 2019, pp. 19-22, 2019, doi:

10.1109/SENSORSNANO44414.2019.8940082.

[3] I. W. Jung, B. Park, J. Provine, R. T. Howe, and O. Solgaard, "Highly sensitive monolithic silicon photonic crystal fiber tip sensor for simultaneous measurement of refractive index and temperature," J. Light. Technol., vol. 29, no. 9, pp. 1367-1374, 2011, doi: 10.1109/JLT.2011.2126018.

[4] J. K. Sahota, N. Gupta, and D. Dhawan, "Fiber Bragg grating sensors for monitoring of physical parameters: a comprehensive review," vol. 59, no. May, pp. 1-35, 2021, doi: 10.1117/1.0E.59.6.060901.

[5] Y. Cho, F. Ahmed, H. Joe, H. Yun, B. Min, and M. B. G. Jun, "Fabrication of a Screw-shaped Long Period Fiber Grating for Refractive Index Sensing," vol. 1135, no. c, 2017, doi: 10.1109/LPT.2017.2765598. 
[6] L. Dinia and F. Frezza, "Laser Beam Self-Focusing in Optical Fiber controlled through FBG integration," 2020 IEEE Int. Work. Metrol. Ind. 4.0 loT, Metrolnd 4.0 IoT 2020 - Proc., pp. 264-267, 2020, doi:

10.1109/Metrolnd4.0loT48571.2020.9138276.

[7] M. Y. Fu, "Refractive index sensing based on the reflectivity of the backward cladding-core mode coupling in a concatenated fiber bragg grating and a long period grating," IEEE Sens. J., vol. 12, no. 5, pp. 1415-1420, 2012, doi: 10.1109/JSEN.2011.2172601.

[8] C. Guan, X. Tian, S. Li, X. Zhong, J. Shi, and L. Yuan, "Long period fiber grating and high sensitivity refractive index sensor based on hollow eccentric optical fiber," Sensors Actuators, B Chem., vol. 188, pp. 768-771, 2013, doi: 10.1016/j.snb.2013.07.086.

[9] R. Yang, Y.-S. Yu, Y. Xue, C. Chen, Q.-D. Chen, and H.-B. Sun, "Single S-tapered fiber Mach-Zehnder interferometers," Opt. Lett., vol. 36, no. 23, p. 4482, 2011, doi: 10.1364/ol.36.004482.

[10] M. Divya shree, A. Sangeetha, and P. Krishnan, "Analysis and optimization of uniform FBG structure for sensing and communication applications," Photonic Netw. Commun., vol. 39, no. 3, pp. 223-231, 2020, doi: 10.1007/s11107-020-00880-1.

[11] F. De-Jun, Z. Mao-Sen, G. Liu, L. Xi-Lu, and J. Dong-Fang, “D-shaped plastic optical fiber sensor for testing refractive index," IEEE Sens. J., vol. 14, no. 5, pp. 1673-1676, 2014, doi: 10.1109/JSEN.2014.2301911.

[12] A. Othonos, K. Kalli, and G. E. Kohnke, " Fiber Bragg Gratings: Fundamentals and Applications in Telecommunications and Sensing," Phys. Today, vol. 53, no. 5, pp. 61-62, 2000, doi: 10.1063/1.883086.

[13] F. Ahmed, V. Ahsani, A. Saad, and M. B. G. Jun, "Bragg grating embedded in Mach-Zehnder interferometer for refractive index and temperature sensing," IEEE Photonics Technol. Lett., vol. 28, no. 18, pp. 1968-1971, 2016, doi: 10.1109/LPT.2016.2580582.

[14] J. Zhao et al., "Refractive Index Fiber Laser Sensor by Using Tunable Filter Based on No-Core Fiber," IEEE Photonics J., vol. 8, no. 5, 2016, doi: 10.1109/JPHOT.2016.2609598.

[15] M. Gu, S. Yuan, Q. Yuan, and Z. Tong, "Temperature-independent refractive index sensor based on fiber Bragg grating and spherical-shape structure," Opt. Lasers Eng., vol. 115, no. November 2018, pp. 8689, 2019, doi: 10.1016/j.optlaseng.2018.11.018.

[16] P. Xue, F. Yu, B. Wu, H. Bao, and J. Zheng, "Investigation of a D-shaped Plastic Optical Fiber Assisted by a Long Period Grating for Refractive Index Sensing," IEEE Sens. J., vol. PP, no. c, p. 1, 2019, doi: 10.1109/JSEN.2019.2944932.

[17] A. ladicicco, A. Cusano, S. Campopiano, A. Cutolo, and M. Giordano, "Thinned fiber Bragg gratings as refractive index sensors," IEEE Sens. J., vol. 5, no. 6, pp. 1288-1294, 2005, doi:

10.1109/JSEN.2005.859288. 
[18] D. L. O. Presti et al., "Fiber Bragg Gratings for Medical Applications and Future Challenges: A Review," IEEE Access, vol. 8, pp. 156863-156888, 2020.

[19] P. Tian et al., "Refractive Index Sensor Based on Fiber Bragg Grating in Hollow Suspended-Core Fiber," IEEE Sens. J., vol. 19, no. 24, pp. 11961-11964, 2019, doi: 10.1109/JSEN.2019.2938786.

[20] C. Liao, K. Yang, J. Wang, Z. Bai, Z. Gan, and Y. Wang, "Helical Microfiber Bragg Grating Printed by Femtosecond Laser for Refractive Index Sensing," IEEE Photonics Technol. Lett., vol. 31, no. 12, pp. 971974, 2019, doi: 10.1109/LPT.2019.2912634.

[21] P. Chen, X. Shu, F. Shen, and H. Cao, "Sensitive refractive index sensor based on an assembly-free fiber multi-mode interferometer fabricated by femtosecond laser," Opt. Express, vol. 25, no. 24, p. 29896, 2017, doi: 10.1364/oe.25.029896.

[22] P. Niu, J. Zhao, C. Zhang, H. Bai, X. Sun, and J. Bai, "Reflective intensity-demodulated refractometer based on S fiber taper," IEEE Photonics Technol. Lett., vol. 30, no. 1, pp. 55-58, 2018, doi:

10.1109/LPT.2017.2773638.

[23] O. Frazao et al., "EDFA gain flattening using long-period fibre gratings based on the electric arc technique," pp. 4-6, 2001, [Online]. Available: http://discovery.ucl.ac.uk/1314010/.

[24] S. Korganbayev et al., "Compact Fiber Optic Sensors for Dual Temperature and Refractive Index Profiling Based on Partially Etched Chirped Fiber Bragg Grating@nu.edu.kz," Proc. IEEE Sensors, vol. 2018-Octob, 2018, doi: 10.1109/ICSENS.2018.8589819.

[25] Z. Zhang, T. Guo, and B. O. Guan, "Reflective fiber-optic refractometer using broadband cladding mode coupling mediated by a tilted fiber bragg grating and an in-fiber mirror," J. Light. Technol., vol. 37, no. 11, pp. 2815-2819, 2019, doi: 10.1109/JLT.2018.2838538.

[26] Y. Singh, A. Sadhu, and S. K. Raghuwanshi, "Fabrication and Experimental Analysis of Reduced Graphene Oxide Coated Etched Fiber Bragg Grating Refractometric Sensor," IEEE Sensors Lett., vol. 4, no. 7, pp. 20-23, 2020, doi: 10.1109/LSENS.2020.3002837.

[27] W. Zhang, D. Webb, and G. Peng, "Polymer optical fiber Bragg grating acting as an intrinsic biochemical concentration sensor," Opt. Lett., vol. 37, no. 8, p. 1370, 2012, doi: 10.1364/ol.37.001370.

[28] S. R. Tahhan, R. Z. Chen, S. Huang, K. I. Hajim, and K. P. Chen, "Fabrication of Fiber Bragg Grating Coating with TiO2 Nanostructured Metal Oxide for Refractive Index Sensor," J. Nanotechnol., vol. 2017, 2017, doi: 10.1155/2017/2791282.

[29] S. Korposh, S. W. Lee, S. W. James, and R. P. Tatam, "Refractive index sensitivity of fibre-optic long period gratings coated with SiO2 nanoparticle mesoporous thin films," Meas. Sci. Technol., vol. 22, no. 7, 2011, doi: 10.1088/0957-0233/22/7/075208. 
[30] Y. Zhao, Y. Liu, C. Zhou, Q. Guo, and T. Wang, "Sensing Characteristics of Long-Period Fiber Gratings Written in Thinned Cladding Fiber," IEEE Sens. J., vol. 16, no. 5, pp. 1217-1223, 2016, doi: 10.1109/JSEN.2015.2501411.

[31] C. Li, W. Hu, L. Ding, and N. Yang, "Simultaneous measurement of refractive index and temperature based on reflective LPG-FBGs," OECC/PSC 2019 - 24th Optoelectron. Commun. Conf. Conf. Photonics Switch. Comput. 2019, vol. 26, no. 21, pp. 2193-2196, 2019, doi: 10.23919/PS.2019.8817732.

[32] Y. Dong, S. Xiao, B. Wu, H. Xiao, and S. Jian, "Refractive index and temperature sensor based on Dshaped fiber combined with a fiber bragg grating," IEEE Sens. J., vol. 19, no. 4, pp. 1362-1367, 2019, doi: 10.1109/JSEN.2018.2880305.

[33] K. O. Hill and G. Meltz, “Fiber Bragg grating technology fundamentals and overview," J. Light. Technol., vol. 15, no. 8, pp. 1263-1276, 1997, doi: 10.1109/50.618320.

[34] H. Chen, C. Tien, W. F. Liu, S. Lin, and C. Engineering, "The Measurement of Liquid Refractive Index by D-shaped Fiber Bragg Grating," pp. 119-120.

[35] R. B. Liang, Q. Z. Sun, J. H. Wo, and D. M. Liu, "Theoretical investigation on refractive index sensor based on Bragg grating in micro/nanofiber," Wuli Xuebao/Acta Phys. Sin., vol. 60, no. 10, pp. 0-2, 2011, doi: $10.7498 / a p s .60 .104221$.

\section{Tables}

Table 1 is not available with this version

\section{Figures}




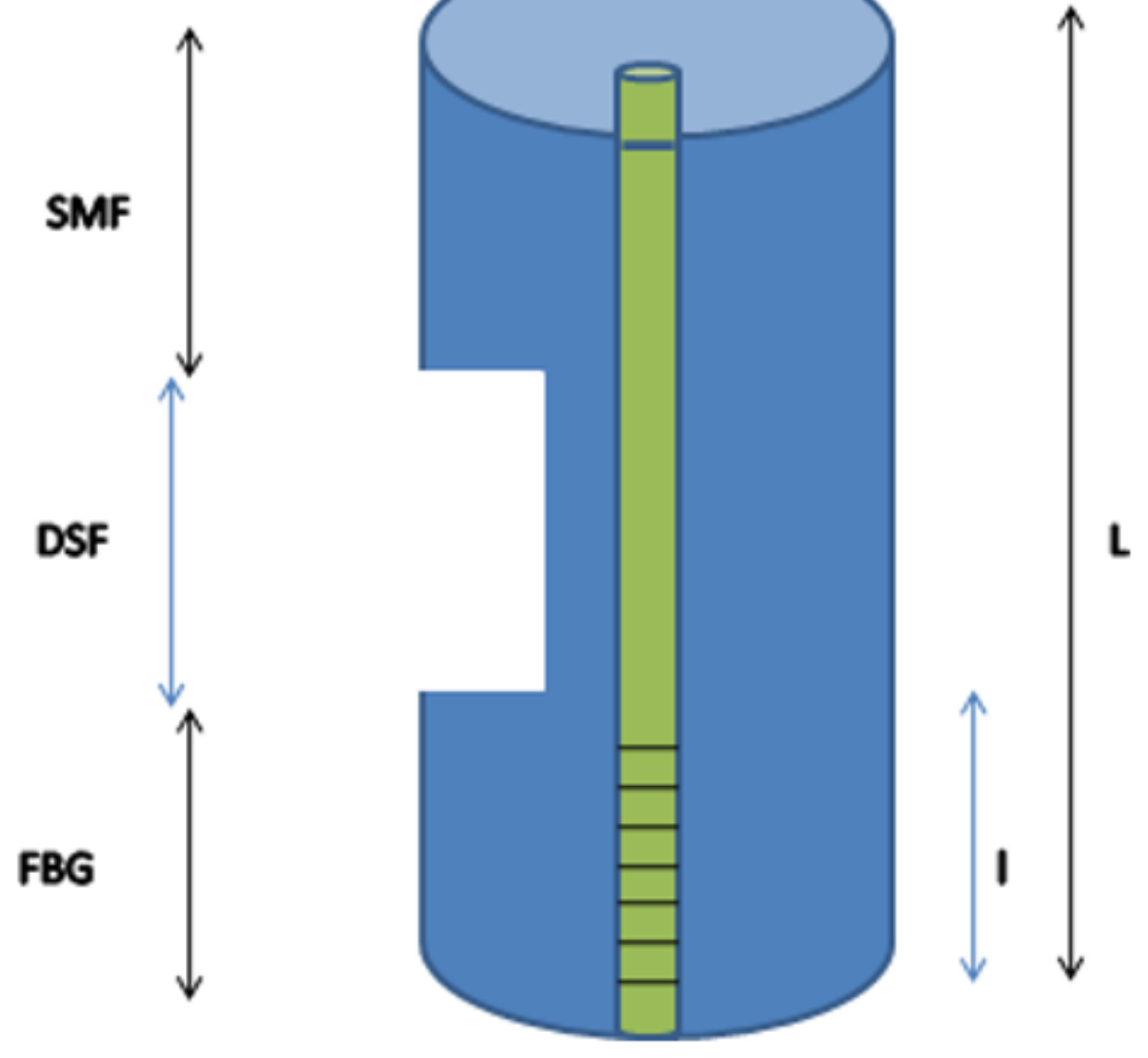

Figure 1

Schematic diagram of $D$-shaped fiber 


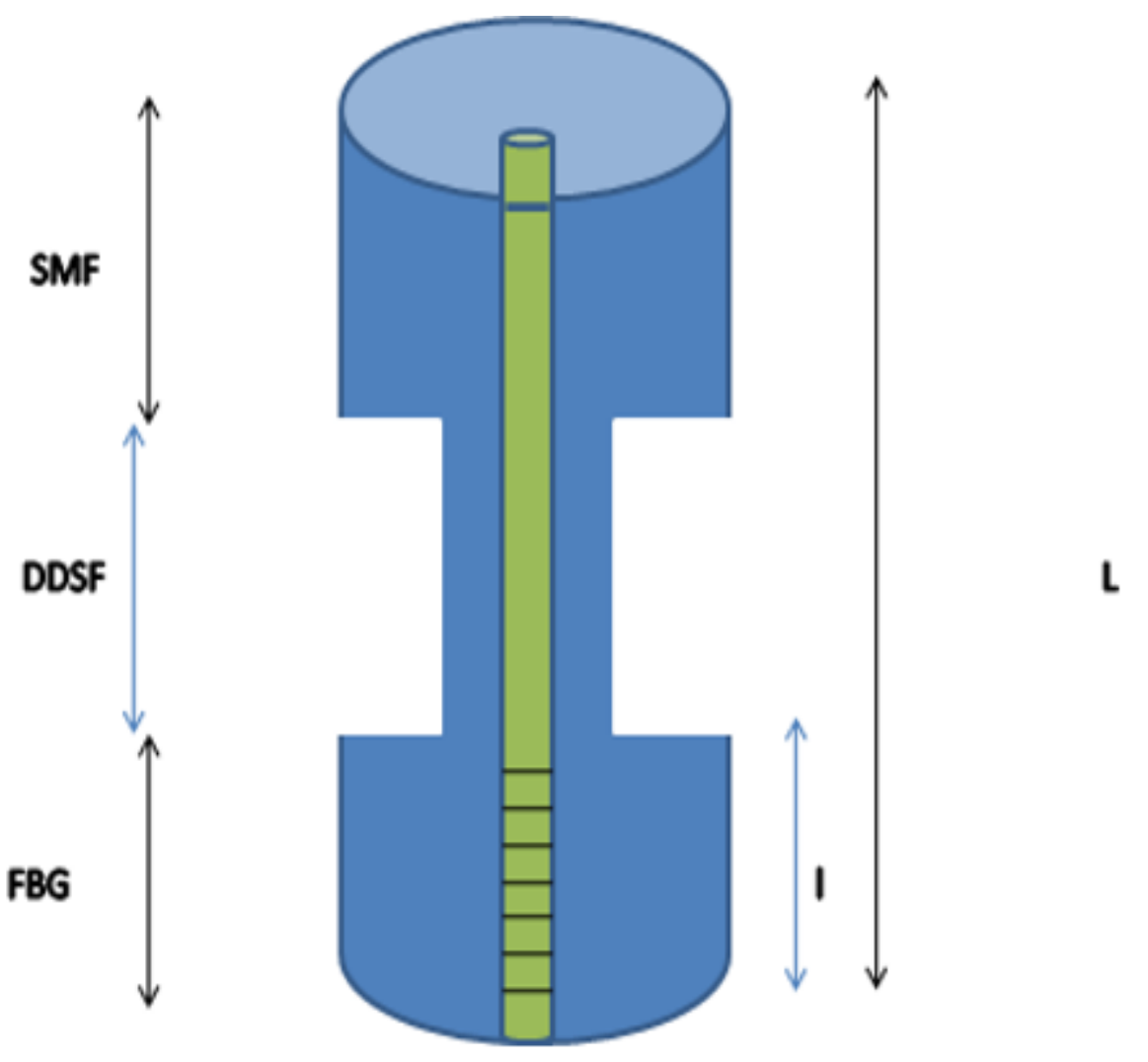

Figure 2

Schematic diagram of double D-shaped fiber 
Contour Map of Index Profile at $Y=0$
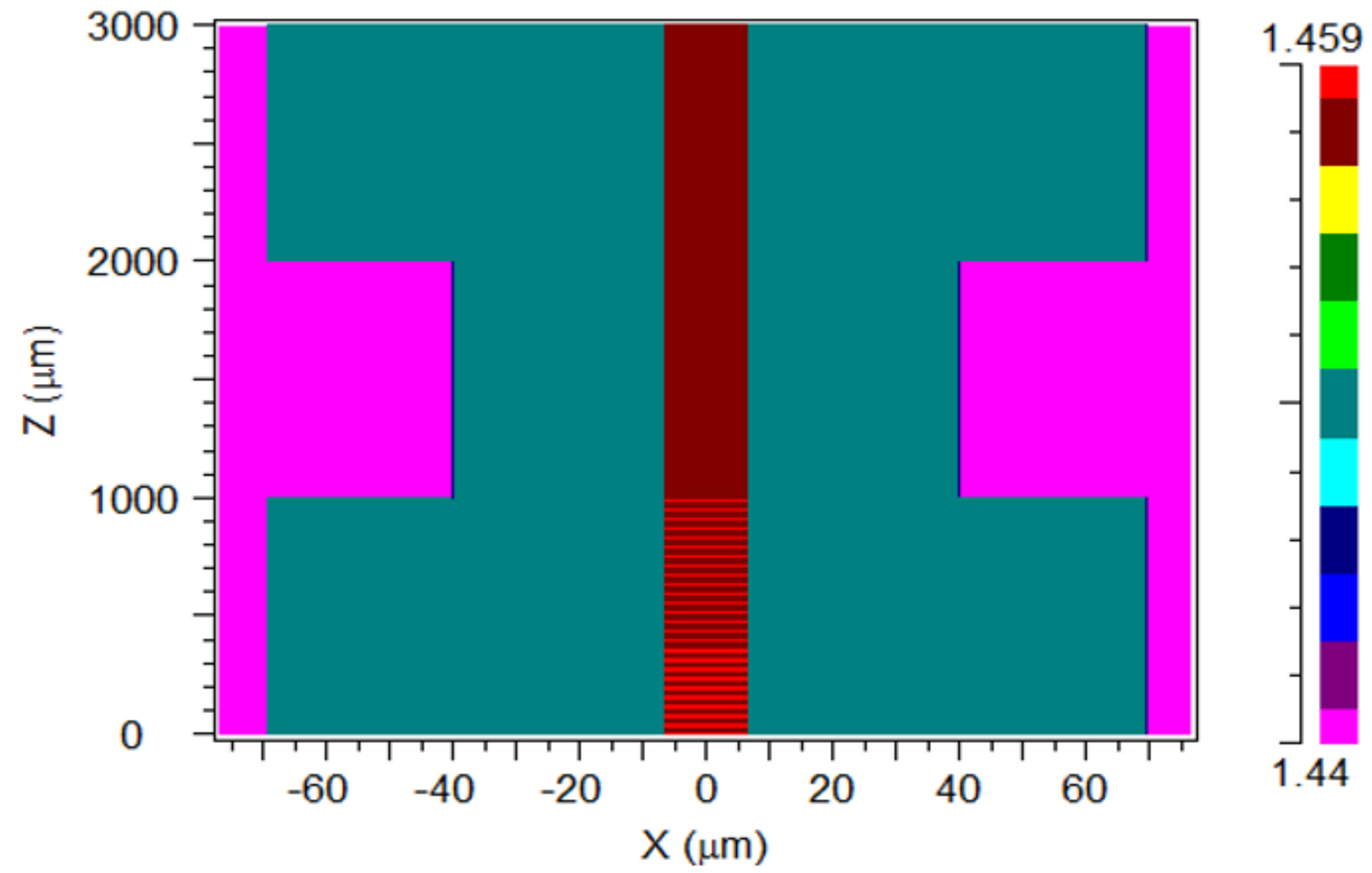

Figure 3

Refractive index distribution of double D-shaped fiber 


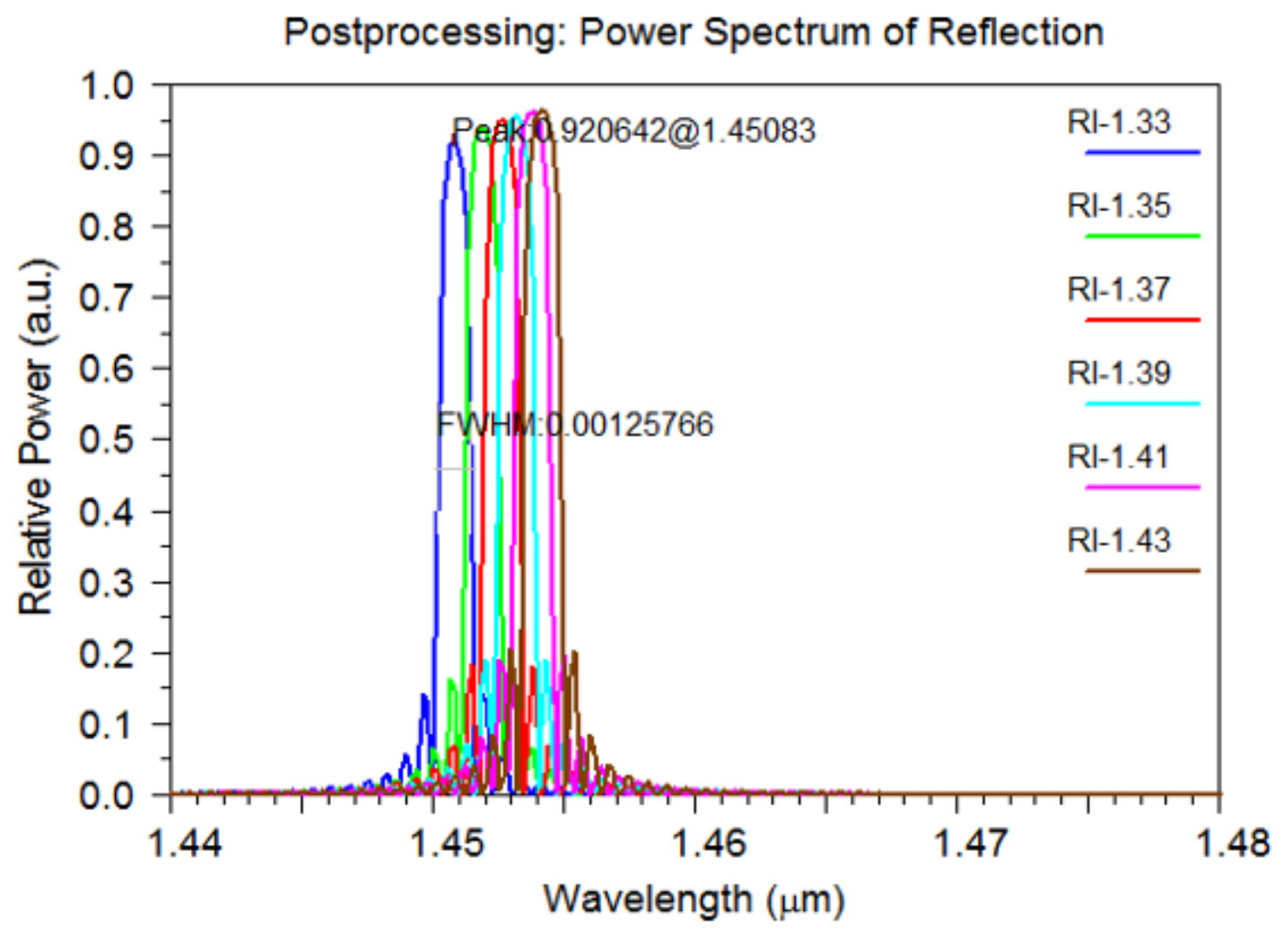

Figure 4

Grating spectral response of DSF for RI ranging from 1.33 to 1.43 


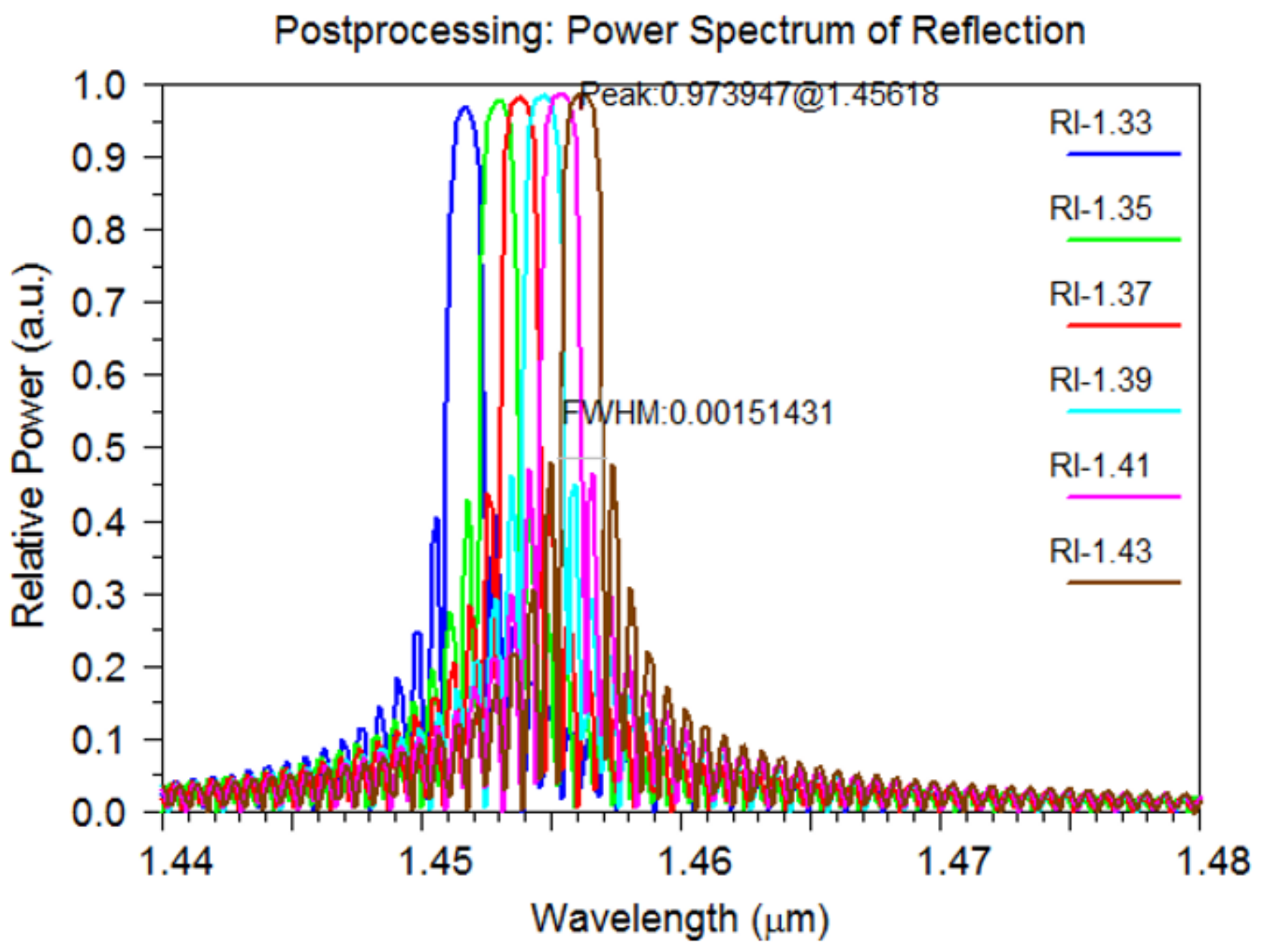

Figure 5

Grating spectral response of DDSF for RI ranging from 1.33 to 1.43 


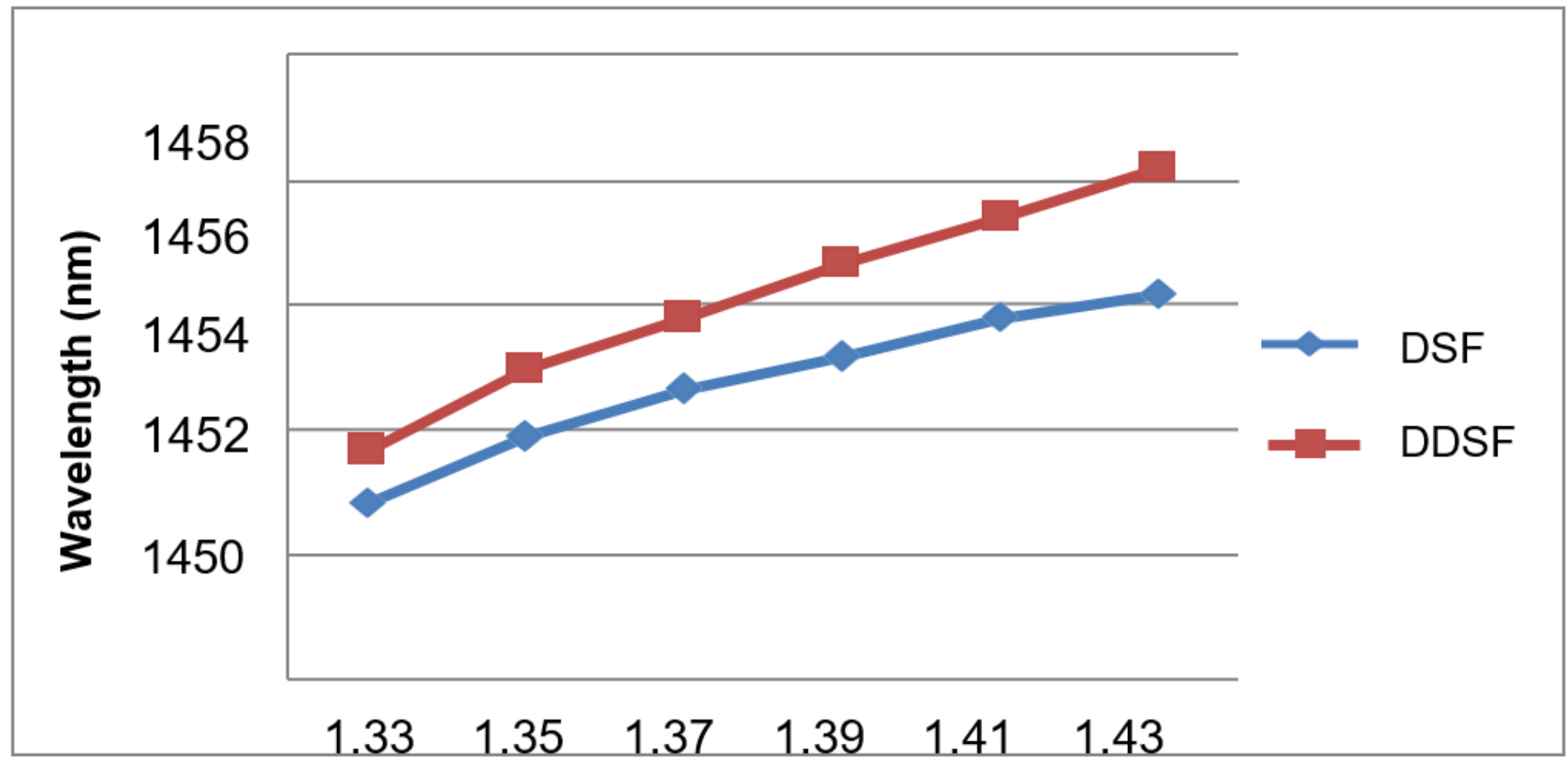

Figure 6

Sensitivity curve for D-shaped and double D-shaped fiber

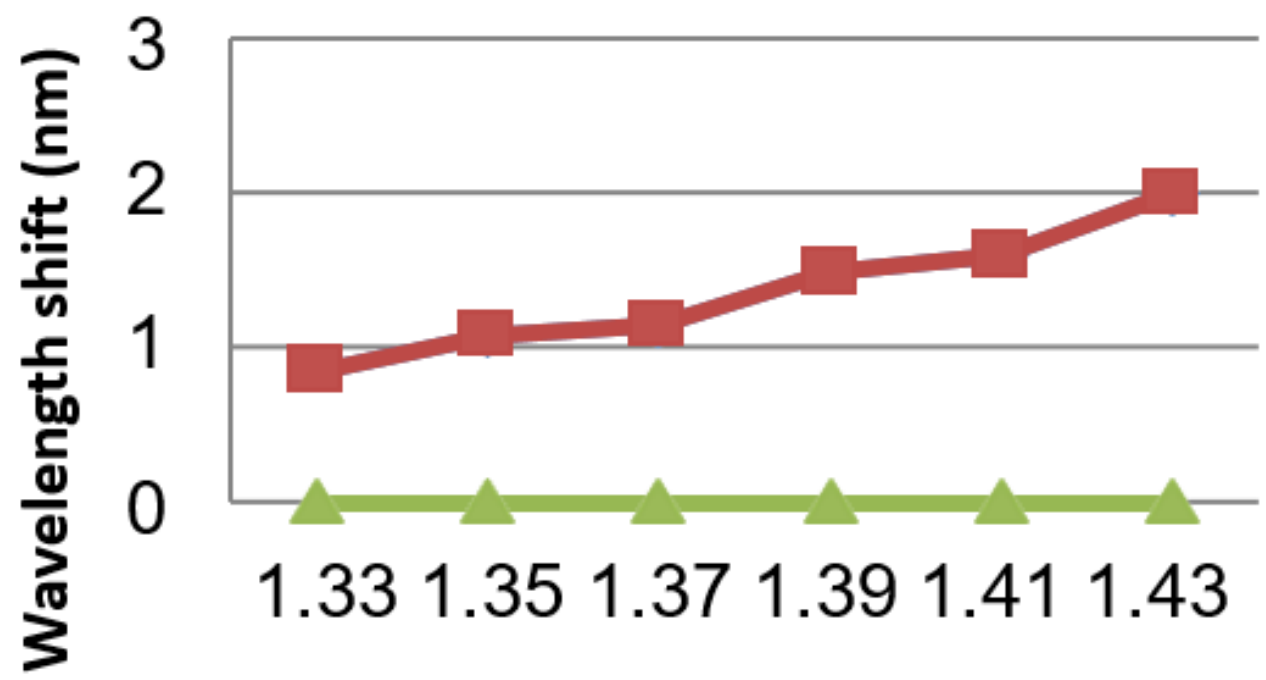

DDSF DSF

Figure 7

Shift of wavelength of D-shaped and double D-shaped fiber versus the refractive index 